\title{
GAUGE INTERPRETATION OF CHARACTERISTIC CLASSES
}

\author{
M. Castrillón López and J. Muñoz Masqué
}

\begin{abstract}
It is proved that characteristic forms generate, over $\Omega^{\bullet}(M)$, the algebra of differential forms on the bundle of connections $p: C(P) \rightarrow M$ of a principal $G$-bundle $\pi: P \rightarrow M$ which are invariant under the natural representation of the gauge algebra of $P$ on connections. The invariance under the Lie algebra of all infinitesimal automorphisms of $P$ is also analyzed.
\end{abstract}

\section{Introduction}

Let $p: C(P) \rightarrow M$ be the bundle of connections of a principal $G$-bundle $\pi: P \rightarrow M$. Let aut $P$ be the Lie algebra of all $G$-invariant vector fields on $P$. We think of aut $P$ as being the "infinitesimal autmorphisms" of $P$. The gauge algebra of $P$, denoted by gau $P$, is the ideal of aut $P$ defined by the $\pi$-vertical vector fields. As the automorphisms of $P$ acts on connections by pulling back conection forms, we obtain a natural Lie algebra homomorphism aut $P \rightarrow \mathfrak{X}(C(P)), X \mapsto X_{C}$. Then, a differential form $\Omega_{r}$ on $C(P)$ is said to be aut $P$-invariant (resp. gauge invariant) if $L_{X_{C}} \Omega_{r}=0$, for every $X \in$ aut $P$ (resp. for every $X \in \operatorname{gau} P)$. The group $G$ acts on $J^{1}(P)$ by setting $j_{x}^{1} s \cdot g=j_{x}^{1}\left(R_{g} \circ s\right)$ and there is a natural identification $J^{1}(P) / G \cong C(P)$ so that the quotient mapping $q: J^{1}(P) \rightarrow C(P)$ is a principal $G$-bundle. The canonical contact form $\theta$ on $J^{1}(P)$ can be considered as a connection form on the principal bundle $q$ (for the details, for example see [4]). Let $\Theta$ be its curvature form. As is well known (e.g., see [9, XII.Th.1.1.(1)]), for every Weil polynomial $f$ the form $f(\Theta)$ is projectable onto $C(P)$. We call each of such forms a "characteristic form" as they induce the characteristic classes of $P$. In fact, as $f(\Theta)$ is a closed $2 d$-form, with $d=\operatorname{deg} f$, and $p: C(P) \rightarrow M$ is an affine bundle, the form $f(\Theta)$ defines a cohomology class in $H^{2 d}(C(P) ; \mathbb{R}) \cong H^{2 d}(M ; \mathbb{R})$. This argument provides a simple proof of Weil's homomorphism theorem [9, Chapter XII, §1], since the independence of the cohomology class of $\sigma_{\Gamma}^{*} f(\Theta)$ with respect to the choice of $\Gamma$, is now evident: This an interesting consequence of working on the bundle of connections.

The main goal of this paper is to prove the following theorem:

Received April 20, 2001.

1991 Mathematics Subject Classification. Primary 53C05; Secondary 17B66, 22E65, 57R20, $81 \mathrm{R} 10$

Key words and phrases. Connections on a principal bundle, characteristic classes, gauge invariance, jet bundles.

Supported by the DGESIC (Spain) under grant no. PB98-0533. 
Theorem 1. If $G$ is connected, for every gauge invariant differential form $\Omega_{r}$ on $C(P)$ there exists differential forms $\omega_{1}, \ldots, \omega_{k}$ and Weil polynomials $f_{1}, \ldots, f_{k}$ such that $\Omega_{r}=p^{*}\left(\omega_{1}\right) \wedge f_{1}(\Theta)+\ldots+p^{*}\left(\omega_{k}\right) \wedge f_{k}(\Theta)$.

For a Weil polynomial we mean an element of the symmetric algebra of $\mathfrak{g}^{*}(\mathfrak{g}$ being the Lie algebra of $G$ ) which is invariant under the coadjoint representation. As a consequence of the above theorem we obtain

Corollary 1. With the same assumptions as in Theorem 1, a differential form on $C(P)$ is aut $P$-invariant if and only if it is of the form $f(\Theta)$ where $f$ is a Weil polynomial. Hence every aut $P$-invariant differential form on $C(P)$ is closed.

If the Weil algebra is finitely generated, i.e., $S^{\bullet}\left(\mathfrak{g}^{*}\right)^{G}=\mathbb{R}\left[f_{1}, \ldots, f_{l}\right]$ (for example, this is the case for a complex semisimple Lie algebra according to a result by Chevalley), then gauge invariant forms are generated by $f_{1}(\Theta), \ldots, f_{l}(\Theta)$ so that these forms are a natural basis of characteristic forms. The standard example is $G=U(n)$ where $f_{i}$ are the coefficients of the characteristic polynomial and $f_{i}(\Theta)$ can be considered as universal Chern forms.

As an example, let us consider a principal $S U(2)$-bundle $\pi: P \rightarrow M$. In this case the Weil polynomials are generated by the determinant det: $\mathfrak{s u}(2) \rightarrow$ $\mathbb{R}$ (e.g. see [9, XII, Theorem 2.5]). We use $-i / 2$ times the Pauli matrices as a basis $B_{1}, B_{2}, B_{3}$ of $\mathfrak{s u}(2)$. If $P$ is trivial over $U \subseteq M$, for every $B \in$ $\mathfrak{s u}(2)$ we define a one-parameter group of gauge transformations on $U$ by setting $\varphi_{t}^{B}(x, g)=(x, \exp (t B) \cdot g)$. Let $\tilde{B}$ be the corresponding infinitesimal generator. Then, $\tilde{B}_{1}, \tilde{B}_{2}, \tilde{B}_{3}$ is a basis of ad $\pi^{-1}(U)$. Let $\sigma_{\Gamma}: M \rightarrow C(P)$ be the section induced by a connection $\Gamma$ on $P$ (see $\S 2.2$ below). There exist unique functions $A_{j}^{a}(\Gamma) \in C^{\infty}(U)$ such that $\sigma_{\Gamma}\left(\partial / \partial x^{j}\right)=\partial / \partial x^{j}-A_{j}^{a}(\Gamma) \tilde{B}_{a}, 1 \leq j \leq n$. The functions $\left(x^{j} ; A_{j}^{a}\right), 1 \leq j \leq n, 1 \leq a \leq 3$, induce a natural coordinate system on $p^{-1}(U)=C\left(\pi^{-1} U\right)$. As a computation shows, the expression of $\operatorname{det}(\Theta)$ in these coordinates is

$$
\operatorname{det}(\Theta)=\frac{1}{4} \mathfrak{S}_{123}\left(\mathrm{~d} A_{i}^{1} \wedge \mathrm{d} x^{i} \wedge \mathrm{d} A_{j}^{1} \wedge \mathrm{d} x^{j}+2 A_{j}^{2} A_{k}^{3} \mathrm{~d} x^{j} \wedge \mathrm{d} x^{k} \wedge \mathrm{d} A_{i}^{1} \wedge \mathrm{d} x^{i}\right),
$$

where $\mathfrak{S}_{123}$ denotes cyclic sum with respect to the three indices $1,2,3$, and we have $\mathcal{I}_{\text {gau } P}=p^{*} \Omega^{\bullet}(M)[\operatorname{det}(\Theta)], \mathcal{I}_{\text {aut } P}=\mathbb{R}[\operatorname{det}(\Theta)]$ (see $\S 3$ for notations). Moreover, the cohomology class of $\operatorname{det}(\Theta)$ in $H^{4}(C(P) ; \mathbb{R})$ coincides with $-4 \pi^{2}$ times $p^{*}\left(c_{2}(P)\right)$, where $c_{2}(P)$ stands for the second Chern class of $P$.

Similarly, for a principal $U(2)$-bundle we have $\mathcal{I}_{\text {gau } P}=p^{*} \Omega^{\bullet}(M)[\operatorname{tr}(\Theta)$, $\operatorname{det}(\Theta)], \mathcal{I}_{\text {aut } P}=\mathbb{R}[\operatorname{tr}(\Theta), \operatorname{det}(\Theta)]$, as the Weil algebra is generated by the trace and the determinant in this case.

Let us consider the general case again. For every Weil polynomial $f \in S^{\bullet}\left(\mathfrak{g}^{*}\right)^{G}$ we have $\sigma_{\Gamma}^{*}(f(\Theta))=f\left(\Omega_{\Gamma}\right)$, where $\Omega_{\Gamma}$ denotes the curvature form of $\Gamma$ but the form $f(\Theta)$ carries more information than the set of forms $f\left(\Omega_{\Gamma}\right)$. For example if $\operatorname{dim} M=n \leq 3$ and $\operatorname{deg} f=d \geq 2$, then the form $f\left(\Omega_{\Gamma}\right)$ on $M$ vanishes identically for every connection $\Gamma$. Nevertheless, if $d \leq m+1, m=\operatorname{dim} G$, the form $f(\Theta)$ does not vanish in general as it is a differential form of degree $2 d$ 
on a manifold of dimension $n(m+1) \geq 2(m+1)$. In fact, if $G=S U(2)$ and $\operatorname{dim} M=n \leq 3$, then $\sigma_{\Gamma}^{*} \operatorname{det}(\Theta)=0$ for every connection $\Gamma$ but $\operatorname{det}(\Theta) \operatorname{does} n o t$ vanish obviously.

The first motivation for the previous results was the geometric formulation of Utiyama's theorem (e.g. see [2], [3], [5]), an outstanding version of the seminal work by Utiyama on minimal coupling. This version classifies the Lagrangian densities on the bundle of connections of a principal bundle, which are invariant under the gauge algebra representation. Because of the role that Utiyama's result has played in gauge theories, it seems of interest to ask for invariant differential forms of arbitrary degree, not only under the gauge group but also under the group of all automorphisms of the given bundle on a purely geometric setting.

The specific link between Theorem 1 and Utiyama's classification is as follows. If $\Omega_{n}$ is a gauge invariant $n$-form on $C(P)$, then the horizontal $n$-form that $\Omega_{n}$ induces on $J^{1}(C(P))$ is a Lagrangian density that remains invariant under the gauge group in the Utiyama sense.

Moreover, Corollary 1 can be useful in classifying variationally trivial natural Lagrangians on the bundle of connections. Remark that, unlike gauge group, invariance under the full Lie algebra of infinitesimal automorphisms leads one to variationally trivial densities: Its action functional is constant and produces some characteristic numbers of the bundle.

Finally, we should remark that Theorem 1 and its corollaries can be used as a general geometric setting in studying characteristic classes from the differential point of view, and more concretely in dealing with invariant theorems like AtiyahSinger index theorem and Gilkey's results [6] on the subject.

\section{Preliminaries}

2.1. Principal bundle automorphisms. Let $\pi: P \rightarrow M$ be a principal bundle with structure group a Lie group $G$. An automorphism of $\pi: P \rightarrow M$ is a diffeomorphism $\Phi: P \rightarrow P$ satisfying $\Phi(u \cdot g)=\Phi(u) \cdot g, \forall u \in P, \forall g \in G$. The set of all automorphisms of $P$ is a group under the composition of maps which will be denoted by Aut $P$. An automorphism $\Phi \in$ Aut $P$ induces a unique diffeomorphism $\phi: M \rightarrow M$ such that $\pi \circ \Phi=\phi \circ \pi$. If $\phi=\operatorname{id}_{M}$, then $\Phi$ is said to be a gauge transformation ( $c f$. [3, 3.2.1], [7, III.35]). The set of all gauge transformations is a subgroup Gau $P \subset$ Aut $P$, which is called the gauge group. Let $R_{g}: P \rightarrow P$ be the right translation by $g \in G$. A vector field $X \in \mathfrak{X}(P)$ is said to be $G$-invariant if $R_{g} \cdot X=X, \forall g \in G$. If $\Phi_{t}$ is the flow of $X$, then $X$ is $G$-invariant if and only if $\Phi_{t} \in$ Aut $P, \forall t \in \mathbb{R}$. Because of this we think of $G$-invariant vector fields as being the "Lie algebra" of the automorphism group Aut $P$ and hence we denote the Lie subalgebra of $G$-invariant vector fields on $P$ by aut $P \subset \mathfrak{X}(P)$. Each $G$-invariant vector field on $P$ is $\pi$-projectable. A $\pi$ vertical vector field $X \in \mathfrak{X}(P)$ is $G$-invariant if and only if $\Phi_{t} \in$ Gau $P, \forall t \in \mathbb{R}$. Accordingly, the ideal of all $\pi$-vertical $G$-invariant vector fields on $P$, called the gauge algebra of $P$, is denoted by gau $P \subset$ aut $P$. 
2.2. Bundle of connections. The group $G$ acts on $T(P)$ by setting $X \cdot g=$ $\left(R_{g}\right)_{*}(X), \forall X \in T(P), \forall g \in G$. The quotient $T(P) / G$ exists and is endowed with a vector bundle structure over $M$ whose global sections can be naturally identified with aut $P$; i.e., aut $P \cong \Gamma(M, T(P) / G)$. The gauge algebra of $P$ can be identified with the global sections of the adjoint bundle; i.e., the bundle $\pi_{\mathfrak{g}}$ : ad $P \rightarrow M$ associated to $P$ by the adjoint representation of $G$ on its Lie algebra $\mathfrak{g}$ (cf. [7, III.35], [9, I.Proposition 5.4]); that is, gau $P \cong \Gamma(M$, ad $P)$. Hence we obtain an exact sequence of vector bundles over $M$ (the so-called Atiyah sequence, [1, Theorem 1]),

$$
0 \rightarrow \operatorname{ad} P \rightarrow T(P) / G \stackrel{\pi_{*}}{\longrightarrow} T M \rightarrow 0 .
$$

The bundle of splittings of the sequence (2.1) is denoted by $p: C(P) \rightarrow M$ and it is called the bundle of connections of $P$ ( $c f$. [5, Definition 4.5]), as connections on $P$ can be identified with the global sections of $p: C(P) \rightarrow M$. If $\Gamma$ is a connection on $P$ and $X^{*} \in \mathfrak{X}(P)$ is the horizontal lift (with respect to $\Gamma$ ) of $X \in \mathfrak{X}(M)$ (cf. $[9$, Ch.II,$\S 1]$ ), then the corresponding splitting is given by $T M \rightarrow T(P) / G$, $X \mapsto X^{*}$. We denote by $\sigma_{\Gamma}: M \rightarrow C(P)$ the section of the bundle of connections tautologically induced by a connection $\Gamma$. We recall that $C(P)$ is an affine bundle modelled over the vector bundle $\operatorname{Hom}(T M, \operatorname{ad} P) \simeq T^{*} M \otimes$ ad $P$.

2.3. Another construction of $C(P)$. Let $\pi_{1}: J^{1} P \rightarrow M$ be the 1-jet bundle of local sections of $\pi: P \rightarrow M$. The group structure $G$ acts (on the right) on $J^{1} P$ by the formula $j_{x}^{1} s \cdot g=j_{x}^{1}\left(R_{g} \circ s\right), j_{x}^{1} s \in J^{1} P, g \in G$. The quotient $\left(J^{1} P\right) / G$ exists as a fibred differentiable manifold over $M$ and can be identified with the bundle of connections. This fact is also used in order to define $C(P)(e . g$., see [2], [8]). This identification is as follows. Each local section $s$ defines a retract $\Gamma_{s(x)}: T_{s(x)} P \rightarrow V_{s(x)} P=\operatorname{ker}\left(\pi_{*}\right)_{s(x)}$ of the inclusion $V_{s(x)} P \subset T_{s(x)} P$ by setting $\Gamma_{s(x)}(X)=X-s_{*} \pi_{*}(X)$. For every $u \in \pi^{-1}(x)$ there exists a unique $g \in G$ such that $u=s(x) \cdot g$ and we define $\Gamma_{u}: T_{u} P \rightarrow V_{u} P$ as $\Gamma_{u}=\left(R_{g}\right)_{*} \circ \Gamma_{s(x)} \circ\left(R_{g^{-1}}\right)_{*}$. In this way we obtain an "element of connection $\Gamma$ at $x$ "; that is, an element of $C(P)$ which only depends on $j_{x}^{1} s$. Hence we define

$$
q: J^{1} P \rightarrow C(P), \quad q\left(j_{x}^{1} s\right)=\Gamma .
$$

It is not difficult to prove that $q$ is a surjective submersion whose fibres are the orbits of $G$; in other words, $q: J^{1} P \rightarrow C(P)$ is a principal $G$-bundle.

\section{Invariance on $C(P)$}

The group Aut $P$ acts (on the left) on connections by $\Gamma \mapsto \Gamma^{\prime}=\Phi(\Gamma)$ for every $\Phi \in$ Aut $P$, where $\Gamma^{\prime}$ is the connection corresponding to the connection form $\omega_{\Gamma^{\prime}}=\left(\Phi^{-1}\right)^{*} \omega_{\Gamma}$ (cf. [9, II.Proposition 6.2-(b)]). If $\Psi \in$ Aut $P$, then $(\Psi \circ \Phi)(\Gamma)=\Psi(\Phi(\Gamma))$. Furthermore, for every $\Phi \in$ Aut $P$ there exists a unique diffeomorphism $\Phi_{C}: C(P) \rightarrow C(P)$ such that $p \circ \Phi_{C}=\phi \circ p$, where $\phi: M \rightarrow M$ is the diffeomorphism induced from $\Phi$, and $\Phi_{C} \circ \sigma_{\Gamma}=\sigma_{\Phi(\Gamma)}$, for every connection $\Gamma$ on $P$. In this way we obtain a group homomorphism Aut $P \rightarrow \operatorname{Diff} C(P)$. If 
$\Phi_{t}$ is the flow of a $G$-invariant vector field $X \in$ aut $P$, then $\left(\Phi_{t}\right)_{C}$ is a oneparameter group on $C(P)$ and the corresponding infinitesimal generator will be denoted by $X_{C}$. In this way we obtain a Lie algebra homomorphism

$$
\text { aut } P \rightarrow \mathfrak{X}(C(P)), \quad X \mapsto X_{C} \text {. }
$$

Notice that $X$ and $X_{C}$ both are projectable onto the same vector field of $M$.

A differential form $\omega_{r}$ on $C(P)$ of degree $r=0, \ldots, n(m+1)=\operatorname{dim} C(P)$, with $n=\operatorname{dim} M, m=\operatorname{dim} G$, is said to be gau $P$-invariant (resp. aut $P$ invariant) if for every $X \in$ gau $P$ (resp. for every $X \in$ aut $P$ ) we have $L_{X_{C}} \omega_{r}=$ 0 . Usually, gau $P$-invariant differential forms are called gauge invariant forms. We denote by $\mathcal{I}_{\text {gau } P}\left(\right.$ resp. by $\mathcal{I}_{\text {aut } P}$ ) the set of gau $P$-invariant differential forms (resp. aut $P$-invariant differential forms). Notice that $\mathcal{I}_{\text {gau } P}$ is a $\mathbb{Z}$-graded algebra over $\Omega^{\bullet}(M)$ and $\mathcal{I}_{\text {aut } P} \subset \mathcal{I}_{\text {gau } P}$ is a subalgebra.

\section{Invariance on $J^{1}(P)$}

4.1. Infinitesimal contact transformations. Let $X$ be a $\pi$-projectable vector field on $P$ and let $X^{\prime}$ be its projection onto $M$. If we denote by $\Phi_{t}, \phi_{t}$ the flows of $X, X^{\prime}$, respectively, then a flow $\Phi_{t}^{(1)}$ can be defined on $J^{1} P$ by setting

$$
\Phi_{t}^{(1)}\left(j_{x}^{1} s\right)=j_{\phi_{t}(x)}^{1}\left(\Phi_{t} \circ s \circ \phi_{-t}\right) .
$$

If $X$ is $\pi$-vertical (i.e., $X^{\prime}=0$ or even $\phi_{t}=\operatorname{id}_{M}$ ) then $\Phi_{t}^{(1)}=J^{1}\left(\Phi_{t}\right)$. We denote by $X^{(1)}$ the infinitesimal generator of the flow $\Phi_{t}^{(1)}$ which is called the infinitesimal contact transformation associated to $X$. We remark that the mapping $X \mapsto X^{(1)}$ is a Lie algebra monomorphism and that $X^{(1)}$ is $\pi_{10}$-projectable onto $X$, where $\pi_{10}: J^{1} P \rightarrow P$ is the canonical projection.

Proposition 1. For every $\Phi \in$ Aut $P$ we have $q \circ \Phi^{(1)}=\Phi_{C} \circ q(c f$. (2.2)). Accordingly, for every $X \in$ aut $P$ the vector field $X^{(1)}$ is q-projectable and its projection is $X_{C}$ (cf. (3.1)).

4.2. The connection form on $J^{1} P$. We define a $\mathfrak{g}$-valued 1 -form $\theta$ on $J^{1} P$ as follows. If $B^{*} \in \mathfrak{X}(P)$ is the fundamental vector field associated to $B \in \mathfrak{g}(c f$. $\left[9\right.$, I.5]) we have an isomorphism $P \times \mathfrak{g} \rightarrow V P$ given by $(u, B) \mapsto B_{u}^{*}$. For every $Y \in T_{j_{x}^{1} s}\left(J^{1} P\right)$ we have $q\left(j_{x}^{1} s\right)\left(\left(\pi_{10}\right)_{*} Y\right) \in V_{s(x)} P(c f .(2.2))$. Accordingly, there exists a unique $B \in \mathfrak{g}$ such that $q\left(j_{x}^{1} s\right)\left(\left(\pi_{10}\right)_{*} Y\right)=B_{s(x)}^{*}$. We set $\theta(Y)=B$. Then, we have $\theta=\theta^{a} \otimes B_{a}$, where $\theta^{a}, 1 \leq a \leq m$, are global ordinary 1-forms on $J^{1} P$ and $B_{1}, \ldots, B_{m}$ is a basis of $\mathfrak{g}$. Let $\left(x^{j}, y^{a}, y_{j}^{a}\right)$ be the coordinates on $J^{1} P$ induced from a fibred coordinate system $\left(x^{j}, y^{a}\right)$ for the projection $\pi: P \rightarrow M$. It is not difficult to see that the forms $\theta^{1}, \ldots, \theta^{m}$ span the same differential system than the standard contact forms $\vartheta^{a}=\mathrm{d} y^{a}-y_{j}^{a} \mathrm{~d} x^{j}, a=1, \ldots, m$. Hence $\left(j^{1} s\right)^{*} \theta^{a}=0$ for every local section $s$.

Proposition 2. The $\mathfrak{g}$-valued 1 -form $\theta$ enjoys the following properties:

(1) For every $\Phi \in$ Gau $P$, we have $J^{1}(\Phi)^{*} \theta=\theta$.

(2) For every $g \in G$, we have $R_{g}^{*} \theta=\operatorname{Ad}_{g^{-1}} \circ \theta$. 
(3) For every $B \in \mathfrak{g}$, let $B^{\bullet}$ be the fundamental vector field associated to $B$ under the action of $G$ on $J^{1} P$. Then $L_{B} \bullet \theta=[\theta, B]$.

(4) For every $B \in \mathfrak{g}$, we have $\theta\left(B^{\bullet}\right)=B$. Hence $\theta$ is a connection form on the principal $G$-bundle $q: J^{1} P \rightarrow C(P)$.

Proof. (1) For every $Y \in T_{j_{x}^{1} s}\left(J^{1} P\right)$ we have $\left(J^{1}(\Phi)^{*} \theta\right)(Y)=\theta\left(J^{1}(\Phi)_{*} Y\right)$ and $\left(\pi_{10}\right)_{*}\left(J^{1}(\Phi)\right)_{*} Y=\left(\pi_{10} \circ J^{1}(\Phi)\right)_{*} Y=\left(\Phi \circ \pi_{10}\right)_{*} Y=\Phi_{*}\left(\left(\pi_{10}\right)_{*} Y\right)$. Hence $\theta\left(J^{1}(\Phi)_{*} Y\right)=C$, where $C \in \mathfrak{g}$ is the element determined by the condition

$$
\Phi_{*}\left[\left(\pi_{10}\right)_{*} Y-s_{*} \pi_{*}\left(\pi_{10}\right)_{*} Y\right]=C_{\Phi(s(x))}^{*} .
$$

Let $B \in \mathfrak{g}$ be the vector defined by $B_{s(x)}^{*}=\left(\pi_{10}\right)_{*} Y-s_{*} \pi_{*}\left(\pi_{10}\right)_{*} Y$. Hence $\theta(Y)=B$, and we have $C_{\Phi(s(x))}^{*}=\Phi_{*} B_{s(x)}^{*}=B_{\Phi(s(x))}^{*}$.

(2) We have

$$
\begin{aligned}
\left(\pi_{10}\right)_{*} J^{1}\left(R_{g}\right)_{*} Y-\left(R_{g} \circ s\right)_{*} \pi_{*}\left(\pi_{10}\right)_{*} J^{1}\left(R_{g}\right)_{*} Y=\left(R_{g}\right)_{*}\left(\pi_{10}\right)_{*} Y \\
-\left(R_{g} \circ s\right)_{*} \pi_{*}\left(R_{g}\right)_{*}\left(\pi_{10}\right)_{*} Y=\left(R_{g}\right)_{*}\left[\left(\pi_{10}\right)_{*} Y-s_{*} \pi_{*}\left(\pi_{10}\right)_{*} Y\right]=\left(R_{g}\right)_{*} B_{s(x)}^{*} \\
=\left(\operatorname{Ad}_{g^{-1}} B\right)^{*}
\end{aligned}
$$

Hence $\left(J^{1}\left(R_{g}\right)^{*} \theta\right)(Y)=\left(\operatorname{Ad}_{g^{-1}} \circ \theta\right)(Y)$.

(3) It follows from (2) taking into account that the flow of $B^{\bullet}$ is $J^{1}\left(R_{\exp (t B)}\right)$.

(4) It follows from the very definition of $\theta$, taking into account that $B^{\bullet}$ is $\pi_{10^{-}}$ projectable onto $B^{*}$.

4.3. Gauge forms on $J^{1} P$. A differential form $\omega_{r}$ on $J^{1} P$ is said to be gauge invariant if $L_{X^{(1)}} \omega_{r}=0$ for all $X \in$ gau $P$.

Remark 1. From Proposition 2-(1) it follows that the forms $\theta^{a}$ are gauge invariant. Hence the components of the curvature form $\Theta=\Theta^{a} \otimes B_{a}$ of $\theta$, are also gauge invariant as $\Theta^{a}=\mathrm{d} \theta^{a}+\frac{1}{2} c_{i j}^{a} \theta^{i} \wedge \theta^{j}$.

Theorem 2. The algebra of gauge invariant forms on $J^{1} P$ is generated by the forms $\left(\theta^{a}, \Theta^{a}\right), 1 \leq a \leq m=\operatorname{dim} G$, over $\pi_{1}^{*} \Omega^{\bullet}(M)$.

Proof. Set $\mathcal{A}^{\prime}(U)=\pi_{1}^{*} \Omega^{\bullet}(U)\left[\theta^{a}, \Theta^{a}\right], 1 \leq a \leq m$, for every open subset $U \subseteq M$. Let $\mathcal{A}(U)$ be the algebra of gauge invariant forms on $J^{1}\left(\pi^{-1} U\right)$. From Remark 1 , we have $\mathcal{A}^{\prime}(U) \subseteq \mathcal{A}(U)$. As $\mathcal{A}^{\prime}$ and $\mathcal{A}$ are sheaves of algebras over $M$, it suffices to prove that $\mathcal{A}^{\prime}(U)=\mathcal{A}(U)$ for every small enough $U$. Hence we can assume that $P$ is trivial, i.e., $P=M \times G$.

Lemma 1. Let $\Omega_{r}$ be a gauge invariant form on $J^{1} P$ and let $s_{0}: M \rightarrow P$ be the unit section: $s_{0}(x)=(x, 1), \forall x \in M$. If $\left(\Omega_{r}\right)_{j_{x}^{1} s_{0}} \in \mathcal{A}_{j_{x}^{1} s_{0}}^{\prime}, \forall x \in M$, then $\left(\Omega_{r}\right)_{j_{x}^{1} s} \in \mathcal{A}_{j_{x}^{1} s}^{\prime}$, for every local section s of $P$.

Proof of Lemma 1. Set $s(x)=(x, \psi(x)), \psi: M \rightarrow G$ being a differentiable mapping and let $\Phi$ be the gauge transformation given by $\Phi(x, g)=(x, \psi(x) g)$. We have $\Phi \circ s_{0}=s$, and since $G$ is connected there exists a one parameter group of gauge transformations $\Phi_{t}$ such that $\Phi_{1}=\Phi$. Let $X$ be the infinitesimal 
generator of $\Phi_{t}$. As $L_{X^{(1)}} \Omega_{r}=0$, we have $J^{1}\left(\Phi_{t}\right)^{*} \Omega_{r}=\Omega_{r}, \forall t \in \mathbb{R}$; in particular for $t=1$. Then we have

$$
\left(\Omega_{r}\right)_{j_{x}^{1} s}=J^{1}\left(\Phi^{-1}\right)^{*}\left(\left(\Omega_{r}\right)_{j_{x}^{1} s_{0}}\right)
$$

and it suffices to take into account that $J^{1}\left(\Phi^{-1}\right)^{*} \mathcal{A}_{j_{x}^{1} s_{0}}^{\prime}=\mathcal{A}_{j_{x}^{1} s}^{\prime}$, in order to finish the proof of the lemma.

According to the lemma, we only need to prove that every gauge invariant form $\Omega_{r}$ belongs to $\mathcal{A}^{\prime}$ along the section $j^{1} s_{0}$.

Let $\left(y^{1}, \ldots, y^{m}\right)$ be the normal coordinates on a neighbourhood of the unit in $G$ associated with the basis $B_{1}, \ldots, B_{m}$ of $\mathfrak{g}$ and let $\left(x^{j}, y^{i} ; y_{j}^{i}\right), 1 \leq i \leq m$, $1 \leq j \leq n$, be the coordinate system induced from $\left(x^{j}, y^{i}\right)$ on $J^{1}(M, G)$; i.e., $y_{j}^{i}\left(j_{x}^{1} s\right)=\left(\partial\left(y^{i} \circ s\right) / \partial x^{j}\right)(x)$. Let us compute the local expression of the forms $\theta^{a}, 1 \leq a \leq m$. As they are contact forms we have $\theta^{a}=f_{i}^{a}\left(\mathrm{~d} y^{i}-y_{j}^{i} \mathrm{~d} x^{j}\right)$, where the functions $f_{i}^{a}$ are seen to be given by $f_{i}^{a}=\left(F^{-1}\right)_{i}^{a}, F=\left(F_{i}^{a}\right)$ being the matrix defined by

$$
F_{i}^{a}=\sum_{\alpha \in \mathbb{N}^{m}} C_{\alpha(i)}^{a}\left(y^{1}\right)^{\alpha_{1}} \cdots\left(y^{m}\right)^{\alpha_{m}}
$$

with $\alpha=\left(\alpha_{1}, \ldots, \alpha_{m}\right) \in \mathbb{N}^{m},(i)=(0, \ldots, 0, \stackrel{(i}{1}, 0, \ldots, 0)$, and $C_{\alpha \beta}^{a}$ are the coefficients in the Baker-Campbell-Hausdorff formula relative to the normal coordinates chosen $(c f .[11,2.15])$. Furthermore, we have

$$
f_{i}^{a}\left(j_{x}^{1} s_{0}\right)=\delta_{i}^{a}, \quad\left(\partial f_{i}^{a} / \partial y^{j}\right)\left(j_{x}^{1} s_{0}\right)=\frac{1}{2} c_{i j}^{a}, \quad\left[B_{i}, B_{j}\right]=c_{i j}^{a} B_{a} .
$$

Hence

$$
\left(\theta^{a}\right)_{j_{x}^{1} s_{0}}=\left(\mathrm{d} y^{i}\right)_{j_{x}^{1} s_{0}}, \quad\left(\mathrm{~d} \theta^{a}\right)_{j_{x}^{1} s_{0}}=\left(\mathrm{d} x^{j} \wedge \mathrm{d} y_{j}^{a}+\frac{1}{2} c_{i j}^{a} \mathrm{~d} y^{j} \wedge \mathrm{d} y^{i}\right)_{j_{x}^{1} s_{0}},
$$

as $y^{a}\left(j_{x}^{1} s_{0}\right)=y_{j}^{a}\left(j_{x}^{1} s_{0}\right)=0$. Taking into account that $\Theta^{a}=\mathrm{d} \theta^{a}+\frac{1}{2} c_{i j}^{a} \theta^{i} \wedge \theta^{j}$, we obtain

$$
\left(\Theta^{a}\right)_{j_{x}^{1} s_{0}}=\left(\mathrm{d} x^{j} \wedge \mathrm{d} y_{j}^{a}\right)_{j_{x}^{1} s_{0}} .
$$

Accordingly, we only need to prove that a gauge invariant form $\Omega_{r}$ can be written as a polynomial in $\theta^{a}, \Theta^{a}$ with coefficients in $\pi_{1}^{*} \Omega^{\bullet}(M)$ along the unit section.

First, we calculate the local expression of $X^{(1)}$ for a given gauge field

$$
X=C_{(b) \alpha}^{i} \lambda^{b}\left(y^{1}\right)^{\alpha_{1}} \cdots\left(y^{m}\right)^{\alpha_{m}} \frac{\partial}{\partial y^{i}},
$$

infinitesimal generator of the flow $\Phi_{t}(x, g)=(x, \exp (t B(x)) \cdot g)$, where $B=\lambda^{i} B_{i}$, $\lambda^{i} \in C^{\infty}(U)$. As standard jet prolongation formulas show (e.g. see [10, $\left.\left.\S 4.4\right]\right)$, by setting $y^{\alpha}=\left(y^{1}\right)^{\alpha_{1}} \cdots\left(y^{m}\right)^{\alpha_{m}}$, we have

$$
X^{(1)}=C_{(b) \alpha}^{i} \lambda^{b} y^{\alpha} \frac{\partial}{\partial y^{i}}+C_{(b) \alpha}^{i}\left(\frac{\partial \lambda^{b}}{\partial x^{j}} y^{\alpha}+\lambda^{b} \alpha_{h} y^{\alpha-(h)} y_{j}^{h}\right) \frac{\partial}{\partial y_{j}^{i}} .
$$


Moreover, we have

$$
\Omega_{r}=f_{H I J} \mathrm{~d} x^{H} \wedge \mathrm{d} y^{I} \wedge\left(\mathrm{d} y_{1}^{1}\right)^{j_{1}^{1}} \wedge \ldots \wedge\left(\mathrm{d} y_{n}^{m}\right)^{j_{n}^{m}}, \quad f_{H I J} \in C^{\infty}\left(J^{1}\left(\pi^{-1} U\right)\right),
$$

with

$$
\mathrm{d} x^{H}=\left(\mathrm{d} x^{1}\right)^{h_{1}} \wedge \ldots \wedge\left(\mathrm{d} x^{n}\right)^{h_{n}}, \quad \mathrm{~d} y^{I}=\left(\mathrm{d} y^{1}\right)^{i_{1}} \wedge \ldots \wedge\left(\mathrm{d} y^{m}\right)^{i_{m}},
$$

where $H=\left(h_{1}, \ldots, h_{n}\right) \in\{0,1\}^{n}, I=\left(i_{1}, \ldots, i_{m}\right) \in\{0,1\}^{m}, J=\left(j_{1}^{1}, \ldots, j_{n}^{m}\right)$ $\in\{0,1\}^{m n}$ are Boolean indices such that $|H|+|I|+|J|=r,|H|$ being the sum $h_{1}+\ldots+h_{n}$ taken in $\mathbb{N}$, and similarly for $|I|,|J|$.

Let us fix an arbitrary point $x_{0} \in U$ and an index $d=1, \ldots, n$. The invariance condition (i.e., $\left.\left(L_{X^{(1)}} \Omega_{r}\right)_{j_{x_{0}}^{1} s_{0}}=0\right)$ for

$$
\lambda^{b}=\frac{1}{2} \delta_{b}^{1}\left(x^{d}-x^{d}\left(x_{0}\right)\right)^{2},
$$

yields

$$
\begin{aligned}
0=\left(f_{H I J} \mathrm{~d} x^{H} \wedge \mathrm{d} y^{I} \wedge\left(\mathrm{d} y_{1}^{1}\right)^{j_{1}^{1}} \wedge \ldots \wedge\left(\mathrm{d} y_{d-1}^{1}\right)^{j_{d-1}^{1}} \wedge\left(\mathrm{d} x^{d}\right)^{j_{d}^{1}} \wedge\right. \\
\left.\quad\left(\mathrm{d} y_{d+1}^{1}\right)^{j_{d+1}^{1}} \wedge \ldots \wedge\left(\mathrm{d} y_{n}^{m}\right)^{j_{n}^{m}}\right)_{j_{x_{0}}^{1} s_{0}} .
\end{aligned}
$$

Hence if $h_{d}=0$ and $j_{d}^{1}=1$, then $f_{H I J}\left(j_{x_{0}}^{1} s_{0}\right)=0$. As $x_{0}$ is arbitrary, we conclude that $\left.f_{H I J}\right|_{j^{1} s_{0}}=0$ whenever an index $d$ exists such that $h_{d}=0$, $j_{d}^{1}=1$. Similarly, for

$$
\lambda^{b}=\delta_{b}^{1}\left(x^{1}-x^{1}\left(x_{0}\right)\right)\left(x^{d}-x^{d}\left(x_{0}\right)\right), \quad 2 \leq d \leq n,
$$

we obtain

$$
\begin{gathered}
0=\left(f_{H I J} \mathrm{~d} x^{H} \wedge \mathrm{d} y^{I} \wedge\left(\mathrm{d} x^{d}\right)^{j_{1}^{1}} \wedge\left(\mathrm{d} y_{2}^{1}\right)^{j_{2}^{1}} \wedge \ldots \wedge\left(\mathrm{d} y_{d-1}^{1}\right)^{j_{n}^{m}}+\right. \\
f_{H I J} \mathrm{~d} x^{H} \wedge \mathrm{d} y^{I} \wedge\left(\mathrm{d} y_{1}^{1}\right)^{j_{1}^{1}} \wedge \ldots \wedge\left(\mathrm{d} y_{d-1}^{1}\right)^{j_{d-1}^{1}} \wedge\left(\mathrm{d} x^{1}\right)^{j_{d}^{1}} \wedge\left(\mathrm{d} y_{d+1}^{1}\right)^{j_{d+1}^{1}} \wedge \\
\left.\ldots \wedge\left(\mathrm{d} y_{n}^{m}\right)^{j_{n}^{m}}\right)_{j_{x_{0}}^{1} s_{0}} .
\end{gathered}
$$

Hence if $J=\left(1, j_{2}^{1}, \ldots, j_{d-1}^{1}, 0, j_{d+1}^{1}, \ldots, j_{n}^{m}\right), \quad \tilde{J}=\left(0, j_{2}^{1}, \ldots, j_{d-1}^{1}, 1\right.$, $\left.j_{d+1}^{1}, \ldots, j_{n}^{m}\right)$ then $f_{H I J}\left(j_{x_{0}}^{1} s_{0}\right)=f_{H I \tilde{J}}\left(j_{x_{0}}^{1} s_{0}\right)$. Accordingly, if $\left.\left(\Omega_{r}\right)\right|_{j^{1} s_{0}}$ contains a summand of the form $\left.\left(\omega_{r-2} \wedge \mathrm{d} x^{d} \wedge \mathrm{d} y_{d}^{1}\right)\right|_{j^{1} s_{0}}$, where $d=1, \ldots, n$ is an arbitrary fixed index, then $\left.\left(\Omega_{r}\right)\right|_{j^{1} s_{0}}$ contains the summand $\left.\left(\omega_{r-2} \wedge \mathrm{d} x^{j} \wedge \mathrm{d} y_{j}^{1}\right)\right|_{j^{1} s_{0}}$. Substituting $i=2, \ldots, m$ succesively for the index 1 in (4.4), (4.5) and recalling the formulas (4.2), (4.3) we have

$$
\begin{aligned}
& \left.\left(\Omega_{r}\right)\right|_{j^{1} s_{0}}= \\
& \left.\quad\left(f_{H I j_{1}, \ldots, j_{m}} \mathrm{~d} x^{H} \wedge\left(\theta^{1}\right)^{i_{1}} \wedge \ldots \wedge\left(\theta^{m}\right)^{i_{m}} \wedge\left(\Theta^{1}\right)^{j_{1}} \wedge \ldots \wedge\left(\Theta^{m}\right)^{j_{m}}\right)\right|_{j^{1} s_{0}} .
\end{aligned}
$$




\section{Proof of Theorem 1}

According to Proposition 1, a differential form $\Omega_{r}$ on $C(P)$ is gauge invariant if and only if $q^{*} \Omega_{r}$ is gauge invariant on $J^{1} P$. Moreover, as $G$ is connected a differential form $\Xi_{r}$ on $J^{1} P$ is $q$-projectable onto $C(P)$ if and only if

$$
\text { 1) } \left.i_{B} \cdot \Xi_{r}=0,2\right) L_{B} \cdot \Xi_{r}=0, \quad \forall B \in \mathfrak{g} .
$$

Hence by virtue of Theorem $2, \mathcal{I}_{\text {gau } P}$ is identified with the subalgebra of $q$ projectable forms in the algebra spanned by $\left\{\theta^{a}, \Theta^{a}\right\}_{1 \leq a \leq m}$ over $\pi_{1}^{*} \Omega^{\bullet}(M)$. Let

$$
\Xi_{r}=\omega_{I, j_{1}, \ldots, j_{m}} \wedge\left(\theta^{1}\right)^{i_{1}} \wedge \ldots \wedge\left(\theta^{m}\right)^{i_{m}} \wedge\left(\Theta^{1}\right)^{j_{1}} \wedge \ldots \wedge\left(\Theta^{m}\right)^{j_{m}},
$$

be a gauge invariant form on $J^{1} P$, with $\omega_{I, j_{1}, \ldots, j_{m}} \in \pi_{1}^{*} \Omega^{r-|I|-2 j}(M), j=$ $j_{1}+\ldots+j_{m}$. By imposing condition 1) in the formula (5.1) and taking into account item (4) in Proposition 2 and that $i_{B} \bullet \Theta=0$ as $\Theta$ is a horizontal form, we obtain

$$
\begin{array}{r}
0=i_{B_{h}^{\bullet}} \Xi_{r}=(-1)^{r-|I|-2 j+i_{1}+\ldots+i_{h-1}} \omega_{I, j_{1}, \ldots, j_{m}} \wedge\left(\theta^{1}\right)^{i_{1}} \wedge \ldots \wedge \widehat{\left(\theta^{h}\right)^{i_{h}}} \wedge \ldots \\
\wedge\left(\theta^{m}\right)^{i_{m}} \wedge\left(\Theta^{1}\right)^{j_{1}} \wedge \ldots \wedge\left(\Theta^{m}\right)^{j_{m}} .
\end{array}
$$

Hence $\omega_{I, j_{1}, \ldots, j_{m}}=0$ for $|I|>0$. Therefore we can rewrite $\Xi_{r}$ as follows:

$$
\Xi_{r}=\omega_{0, j_{1}, \ldots, j_{m}} \wedge\left(\Theta^{1}\right)^{j_{1}} \wedge \ldots \wedge\left(\Theta^{m}\right)^{j_{m}} .
$$

Let $\pi_{1}^{*} \Omega^{\bullet}(M)[\Theta]=\pi_{1}^{*} \Omega^{\bullet}(M)\left[\Theta^{1}, \ldots, \Theta^{m}\right]$ be the algebra of polynomials in the components of the curvature form with coefficients in $\pi_{1}^{*} \Omega^{\bullet}(M)$ and let

$$
E: \pi_{1}^{*} \Omega^{\bullet}(M) \otimes S^{\bullet}\left(\mathfrak{g}^{*}\right) \rightarrow \pi_{1}^{*} \Omega^{\bullet}(M)[\Theta]
$$

be the unique $\mathbb{R}$-linear mapping such that $E(\omega \otimes f)=\omega \wedge f(\Theta)$ for all $\omega \in$ $\pi_{1}^{*} \Omega^{\bullet}(M), f \in S^{\bullet}\left(\mathfrak{g}^{*}\right)$, where $S^{\bullet}\left(\mathfrak{g}^{*}\right)$ denotes the symmetric algebra of $\mathfrak{g}^{*}$. The group $G$ acts on the right on $\pi_{1}^{*} \Omega^{\bullet}(M) \otimes S^{\bullet}\left(\mathfrak{g}^{*}\right)$ and $\pi_{1}^{*} \Omega^{\bullet}(M)[\Theta]$ by setting respectively,

$$
(\omega \otimes f) \cdot g=\omega \otimes f^{g}, \quad \forall \omega \in \pi_{1}^{*} \Omega^{\bullet}(M), \forall f \in S^{k}\left(\mathfrak{g}^{*}\right),
$$

where $f^{g} \in S^{k}\left(\mathfrak{g}^{*}\right)$ is the polynomial defined by

$$
f^{g}\left(v_{1}, \ldots, v_{k}\right)=f\left(\operatorname{Ad}_{g} v_{1}, \ldots, \operatorname{Ad}_{g} v_{k}\right), \quad v_{1}, \ldots, v_{k} \in \mathfrak{g},
$$

and

$$
\Xi \cdot g=\left(R_{g^{-1}}\right)^{*} \Xi, \quad \forall \Xi \in \pi_{1}^{*} \Omega^{\bullet}(M)[\Theta] .
$$

Remark that $\Xi \cdot g \in \pi_{1}^{*} \Omega^{\bullet}(M)[\Theta]$ as $\Theta$ is a differential form of the adjoint type. It is easily checked that $E$ is a $G$-equivariant homogeneous $\mathbb{Z}$-graded algebra epimorphism of degree zero. Set $K=\oplus_{l \geq 0} K^{l}=\operatorname{ker} E$. Assume for the moment the following

Lemma 2. The ideal $K$ is generated by the elements of the form $\omega_{r} \otimes f^{k}$ with $\omega_{r} \in \pi_{1}^{*} \Omega^{r}(M), f^{k} \in S^{k}\left(\mathfrak{g}^{*}\right)$ and $r+k>n=\operatorname{dim} M$. 
First, we remark that, as $G$ is connected, the condition 2) in formula (5.1) is equivalent to saying that $\left(R_{g}\right)^{*} \Xi_{r}=\Xi_{r}, \forall g \in G$. Hence

$$
\mathcal{I}_{\text {gau } P} \cong \pi_{1}^{*} \Omega^{\bullet}(M)[\Theta]^{G}=\left\{\Xi \in \pi_{1}^{*} \Omega^{\bullet}(M)[\Theta]: \Xi \cdot g=\Xi, \forall g \in G\right\} .
$$

Given a homogeneous element $\Xi_{r} \in \mathcal{I}_{\text {gau } P}$, according to Lemma 2 , we can write it as

$$
\Xi_{r}=\omega_{0, j_{1}, \ldots, j_{m}} \wedge\left(\Theta^{1}\right)^{j_{1}} \wedge \ldots \wedge\left(\Theta^{m}\right)^{j_{m}}, \operatorname{deg} \omega_{0, j_{1}, \ldots, j_{m}}+j_{1}+\ldots+j_{m} \leq n .
$$

Note that $r=\operatorname{deg} \omega_{0, j_{1}, \ldots, j_{m}}+2\left(j_{1}+\ldots+j_{m}\right)$. Let $\zeta_{r} \in \pi_{1}^{*} \Omega^{\bullet}(M) \otimes S^{\bullet}\left(\mathfrak{g}^{*}\right)$ be the element defined by

$$
\zeta_{r}=\omega_{0, j_{1}, \ldots, j_{m}} \otimes\left(B_{1}^{*}\right)^{j_{1}} \vee \ldots \vee\left(B_{m}^{*}\right)^{j_{m}},
$$

where $\left(B_{i}^{*}\right)$ is the dual basis of $\left(B_{i}\right)$ and $\vee$ stands for the symmetric product. Hence $E\left(\zeta_{r}\right)=\Xi_{r}$. Then, $\zeta_{r}-\zeta_{r} \cdot g \in K$ for every $g \in G$ and by virtue of Lemma 2 we conclude that $\zeta_{r}-\zeta_{r} \cdot g=0$. Taking into account that $G$ operates trivially on $\pi_{1}^{*} \Omega^{\bullet}(M)$, we have $\left(\pi_{1}^{*} \Omega^{\bullet}(M) \otimes S^{\bullet}\left(\mathfrak{g}^{*}\right)\right)^{G}=\pi_{1}^{*} \Omega^{\bullet}(M) \otimes\left(S^{\bullet}\left(\mathfrak{g}^{*}\right)\right)^{G}$, thus finishing the proof.

5.1. Proof of Lemma 2. We only need to consider the case of an open subset $U \subseteq M$ trivializing $P$. If $\zeta_{l} \in \pi_{1}^{*} \Omega^{\bullet}(M) \otimes S^{\bullet}\left(\mathfrak{g}^{*}\right)$ is an element of degree $l$, then

$$
\zeta_{l}=\omega_{j_{1}, \ldots, j_{m}} \otimes\left(B_{1}^{*}\right)^{j_{1}} \vee \ldots \vee\left(B_{m}^{*}\right)^{j_{m}}, \quad \omega_{j_{1}, \ldots, j_{m}} \in \pi_{1}^{*} \Omega^{l-\left(j_{1}+\ldots+j_{m}\right)}(M),
$$

and we have $E\left(\zeta_{l}\right)=\omega_{j_{1}, \ldots, j_{m}} \wedge\left(\Theta^{1}\right)^{j_{1}} \wedge \ldots \wedge\left(\Theta^{m}\right)^{j_{m}}$. From the formula (4.3) we obtain

$$
\left.\left(E\left(\zeta_{l}\right)\right)\right|_{j^{1} s_{0}}=\left.\omega_{j_{1}, \ldots, j_{m}} \wedge\left(\left(\mathrm{d} x^{i} \wedge \mathrm{d} y_{i}^{1}\right)^{j_{1}} \wedge \ldots \wedge\left(\mathrm{d} x^{i} \wedge \mathrm{d} y_{i}^{m}\right)^{j_{m}}\right)\right|_{j^{1} s_{0}},
$$

where $s_{0}: U \rightarrow U \times G$ is the unit section. By virtue of the formula (4.1) in the proof of Lemma 1, a differential form in $\pi_{1}^{*} \Omega^{\bullet}(M)[\Theta]$ vanishes if and only if it vanishes along $j^{1} s_{0}$. Hence from the formula (5.2) it follows $E\left(\zeta_{l}\right)=0$ for $l>n$. Assume $E\left(\zeta_{l}\right)=0$ and $l \leq n$. In this case, from (5.2) we conclude that for every multi-index $j_{1}, \ldots, j_{m}$ we have

$$
\left.\omega_{j_{1}, \ldots, j_{m}} \wedge\left(\left(\mathrm{d} x^{i} \wedge \mathrm{d} y_{i}^{1}\right)^{j_{1}} \wedge \ldots \wedge\left(\mathrm{d} x^{i} \wedge \mathrm{d} y_{i}^{m}\right)^{j_{m}}\right)\right|_{j^{1} s_{0}}=0
$$

(no summation on $j$ 's).

Set $\omega_{j_{1}, \ldots, j_{m}}=\lambda_{h_{1}, \ldots, h_{j}}^{j_{1}, \ldots, j_{m}} \mathrm{~d} x^{h_{1}} \wedge \ldots \wedge \mathrm{d} x^{h_{j}}$, with $j=l-\left(j_{1}+\ldots+j_{m}\right), h_{1}<\ldots<h_{j}$, and $\lambda_{h_{1}, \ldots, h_{j}}^{j_{1}, \ldots, j_{m}} \in C^{\infty}(U)$. Hence

$$
\left.\lambda_{h_{1}, \ldots, h_{j}}^{j_{1}, \ldots, j_{m}} \mathrm{~d} x^{h_{1}} \wedge \ldots \wedge \mathrm{d} x^{h_{j}} \wedge\left(\left(\mathrm{d} x^{i} \wedge \mathrm{d} y_{i}^{1}\right)^{j_{1}} \wedge \ldots \wedge\left(\mathrm{d} x^{i} \wedge \mathrm{d} y_{i}^{m}\right)^{j_{m}}\right)\right|_{j^{1} s_{0}}=0 .
$$

Once the indices $h_{1}<\ldots<h_{j}$ have been fixed, as $l \leq n$, the expansion of the above formula includes a unique summand of the form

$$
\left.\lambda_{h_{1}, \ldots, h_{j}}^{j_{1}, \ldots, j_{m}} \mathrm{~d} x^{h_{1}} \wedge \ldots \wedge \mathrm{d} x^{h_{j}} \wedge\left(\mathrm{d} x^{i_{1}} \wedge \mathrm{d} y_{i_{1}}^{a_{1}} \wedge \ldots \wedge \mathrm{d} x^{i_{l-j}} \wedge \mathrm{d} y_{i_{l-j}}^{a_{l-j}}\right)\right|_{j^{1} s_{0}},
$$

where $i_{1}<\ldots<i_{l-j}$ is contained in $\{1, \ldots, n\}-\left\{h_{1}, \ldots, h_{j}\right\}$ and the indices $a_{1}, \ldots, a_{l-j}$ run from 1 to $m$. Therefore $\lambda_{h_{1}, \ldots, h_{j}}^{j_{1}, \ldots, j_{m}}=0$ and thus $\zeta_{l}=0$. 
5.2. Proof of Corollary 1. It suffices to prove that the subalgebra of aut $P$ invariant forms in $\pi_{1}^{*} \Omega^{\bullet}(M)[\Theta]$ is $\mathbb{R}[\Theta]=\mathbb{R}\left[\Theta^{1}, \ldots, \Theta^{m}\right]$ as in this case, with the above notations, we have $\mathcal{I}_{\text {aut } P}=\mathbb{R}[\Theta]^{G}=E\left(S^{\bullet}\left(\mathfrak{g}^{*}\right)^{G}\right)$. We prove below that $\theta^{1}, \ldots, \theta^{m}$ (and hence $\left.\Theta^{1}, \ldots, \Theta^{m}\right)$ are aut $P$-invariant. If $\Xi_{r}=\pi_{1}^{*} \omega_{j_{1}, \ldots, j_{m}} \wedge$ $\left(\Theta^{1}\right)^{j_{1}} \wedge \ldots \wedge\left(\Theta^{m}\right)^{j_{m}}$, with $\operatorname{deg} \omega_{j_{1}, \ldots, j_{m}}+j_{1}+\ldots+j_{m} \leq n$ (cf. Lemma 2), is aut $P$-invariant, then for every $X \in$ aut $P$ we have

$$
L_{X_{C}} \Xi_{r}=\pi_{1}^{*}\left(L_{X^{\prime}} \omega_{j_{1}, \ldots, j_{m}}\right) \wedge\left(\Theta^{1}\right)^{j_{1}} \wedge \ldots \wedge\left(\Theta^{m}\right)^{j_{m}}=0,
$$

where $X^{\prime}$ is the projection of $X$ onto the base manifold $M$. Hence $\pi_{1}^{*}\left(L_{X^{\prime}} \omega_{j_{1}, \ldots, j_{m}}\right) \otimes\left(B_{1}^{*}\right)^{j_{1}} \vee \ldots \vee\left(B_{m}^{*}\right)^{j_{m}} \in$ ker $E$ and again by virtue of Lemma 2 we conclude that $L_{X^{\prime}} \omega_{j_{1}, \ldots, j_{m}}=0$ for all $X^{\prime} \in \mathfrak{X}(M)$, so that each $\omega_{j_{1}, \ldots, j_{m}}$ is a constant.

In order to prove that $\theta^{a}$ is aut $P$-invariant we first remark that we only need to prove that $L_{X_{C}} \theta^{a}=0$ for every $X \in \mathfrak{X}(U), U \subseteq M$ being a coordinate open subset such that $\pi^{-1} U \cong U \times G$, where we have identified

$$
X=f^{i} \frac{\partial}{\partial x^{i}}, \quad f^{i} \in C^{\infty}(U)
$$

to the vector field $(X, 0)$ on $U \times G$. By considering a normal coordinate system on $G$ as in the proof of Theorem 2, it is not difficult to check that the local expression for $X^{(1)}$ is the following:

$$
X^{(1)}=f^{i} \frac{\partial}{\partial x^{i}}-y_{i}^{h} \frac{\partial f^{i}}{\partial x^{j}} \frac{\partial}{\partial y_{j}^{h}} .
$$

According to the formula obtained in the proof of Theorem 2, we have $\theta^{a}=$ $f_{i}^{a}\left(\mathrm{~d} y^{i}-y_{j}^{i} \mathrm{~d} x^{j}\right)$ where $f_{i}^{a} \in C^{\infty}(G)$ and the result follows taking into account that $L_{X^{(1)}}\left(\mathrm{d} y^{i}-y_{j}^{i} \mathrm{~d} x^{j}\right)=0$.

\section{References}

[1] M. F. Atiyah, Complex analytic connections in fibre bundles, Trans. Amer. Math. Soc. 85 (1957), 181-207.

[2] D. Betounes, The geometry of gauge-particle field interaction: a generalization of Utiyama's theorem, J. Geom. Phys. 6 (1989), 107-125.

[3] D. Bleecker, Gauge Theory and Variational Principles, Addison-Wesley Publishing Company, Inc., Reading, MA, 1981.

[4] M. Castrillón López and J. Muñoz Masqué, The geometry of the bundle of connections, Math. Z. 236 (2001), 797-811.

[5] D. J. Eck, Gauge-natural bundles and generalized gauge theories, Mem. Amer. Math. Soc. 33 (1981)

[6] P. B. Gilkey, Invariance Theory, the Heat Equation, and the Atiyah-Singer Index Theorem, CRC Press, Boca Raton, FL, 1995.

[7] V. Guillemin and S. Sternberg, Symplectic techniques in physics, Cambridge University Press, Cambridge, 1983.

[8] M. Keyl, About the geometric structure of symmetry-breaking, J. Math. Phys. 32 (1991), $1065-1071$.

[9] S. Kobayashi and K. Nomizu, Foundations of Differential Geometry Volume I and Volume II, John Wiley \& Sons, Inc. (Interscience Division), New York, 1963, 1969. 
[10] D. J. Saunders, The Geometry of Jet Bundles, Cambridge University Press, Cambridge, UK, 1989.

[11] V.S. Varadarajan, Lie groups, Lie algebras, and their representations, Prentice Hall, Inc., N. J., 1974.

Departamento de Geometría y Topología, Facultad de Matemáticas, Universidad Complutense, 28040-Madrid, Spain.

E-mail address: mcastri@mat.ucm.es

CSiC, Instituto de Física Aplicada, C/ Serrano 144, 28006-Madrid, Spain.

E-mail address: jaime@iec.csic.es 